Journal of Mathematics and Statistics 3 (4): 222-227, 2007

ISSN 1549-3644

(C) 2007 Science Publications

\title{
On Discrete Least Squares Polynomial Fit, Linear Spaces and Data Classification
}

\author{
François Dubeau and Youness Mir \\ Département de mathématiques,Université de Sherbrooke, 2500 Boul. de l'Université, \\ Sherbrooke (Qc), Canada, J1K2R1
}

\begin{abstract}
The best discrete least squares polynomial fit to a data set is revisited. We point out some properties related to the best polynomial and precise the dimension of vector spaces encountered to solve the problem. Finally, we suggest a basic classification of data sets based on their increasing or decreasing trend, and on their convexity or concavity form.
\end{abstract}

Keywords: Polynomial data fitting, weighted least squares, orthogonal polynomials, linear spaces, data classification.

\section{INTRODUCTION}

Let $\left\{\left(\omega_{i}, t_{i}, f_{i}\right)_{i=1}^{m}\right.$ be a set of $m$ data points where the $t_{i}$ 's represent the distinct values of the independent variable, the $f_{i}$ 's are the values of the measured function, and each $\omega_{i}$ is the weight associated to the data $\left(t_{i}, f_{i}\right)$. The problem we consider is to find a polynomial $p_{n}$ of degree at most $n$ to fit the data. To measure how well the polynomial fit the data we use the weighted least squares deviation given by

$$
F\left(p_{n}\right)=\sum_{i=1}^{m} \omega_{i}\left(f_{i}-p_{n}\left(t_{i}\right)\right)^{2} .
$$

The best polynomial, called the weighted least squares estimate (WLSE), is given by

$$
p_{n}^{*}=\operatorname{argmin}_{p_{n} \in P_{n}} F\left(p_{n}\right) .
$$

where $P_{n}$ is the set of polynomials of degree at most $n$.

The motivation for this short note comes from a mistake in the proof of Theorem 1 in ${ }^{[5]}$ and explained in the Remark 2 below. The goal of this paper is to clarify the dimension of some vector spaces encountered in solving this problem, establish a property useful for proving the existence of a WLSE for exponential models ${ }^{[2]}$, and suggest a way to classify data using the best polynomial fits. For a standard presentation of the theory related to best (polynomial) least squares fit see ${ }^{[1,3,7,8,9]}$.
The best polynomial fit problem can be solved by considering an orthogonal projection onto $P_{n}$ or, equivalently, by considering an orthogonal projection onto a subspace of $I R^{m}$. In Section 2 we briefly review the solution of the problem in $P_{n}$ and specify the dimension of subspaces of polynomials. In the first part of the Section 3 we consider the subspaces of $I R^{m}$ that play a role in solving the problem in $I R^{m}$. In the second part of this Section 3 we solve the problem using a projection onto a subspace of $I R^{m}$. Finally in Section 4 we suggest a way to classify data which will be useful in the problem of finding existence results for weighted least squares estimator ${ }^{[2]}$.

\section{POLYNOMIAL WEIGHTED LEAST SQUARES FITTING IN $P_{n}$}

In the first part of this section we present the underlying subspaces of $P=\operatorname{Lin}\left\{t^{j} \mid j=0,1,2, \ldots\right\}$ related to the polynomial weighted least squares problem. In the second part we solve the problem using a projection onto a subspace of $P$.

Vector spaces: Let us recall that $P_{n}=\operatorname{Lin}\left\{t^{j} \mid j=0,1, \ldots n\right\}$. We consider also the following two other polynomial subspaces

$$
P V_{k}^{+}=\operatorname{Lin}\left\{v_{k i}^{+}(t)=\left(t+t_{i}\right)^{k} \mid i=1, \ldots m\right\} \subseteq P_{k},
$$

Corresponding Author: $\quad$ François Dubeau, Département de mathématiques Faculté des sciences, Université de Sherbrooke, 2500 Boul. de l'Université, Sherbrooke (Qc), Canada, J1K2R1. Tel: +819821-8000 (62853) Fax: +819-821-7189 


$$
P V_{k}^{-}=\operatorname{Lin}\left\{v_{k \dot{p}}^{-}(t)=\left(t-t_{i}\right)^{k} \mid i=1, \ldots m\right\} \subseteq P_{k},
$$

for any nonnegative integer $k=0,1,2, \ldots$ The next two results specify the dimension of these subspaces.

Theorem 1: Let $P_{n}=\operatorname{Lin}\left\{t^{j} \mid j=0, \ldots, n\right\} \subseteq P$, then $\operatorname{dim} P_{n}=n+1$.

Theorem 2: Let $k$ be any nonnegative integer and let $P V_{k}^{+}$and $P V_{k}^{-}$be defined by (3) and (4).

(a) If $k \leq m-1$ then $P V_{k}^{+}=P_{k}=P V_{k}^{-}$, and $\operatorname{dim} P V_{k}^{+}=k+1=\operatorname{dim} P V_{k}^{-}$.

(b) If $k \geq m$ then $P V_{k}^{+} \subset P_{k}, P V_{k} \subset P_{k} \quad$ and $\operatorname{dim} P V_{k}^{+}=m=\operatorname{dim} P V_{k}$.

Proof: We prove the result for $P V_{k}^{+}$only, the proof for $P V_{k}^{-}$is identical. Since

$$
\sum_{i=1}^{m} \mu_{i} v_{k, i}^{+}(t)=\sum_{i=1}^{m} \mu_{i}\left(\sum_{j=0}^{k}\left(\begin{array}{l}
k \\
j
\end{array}\right) t_{i}^{j} t^{k-j}\right)=\sum_{j=0}^{k}\left(\begin{array}{l}
k \\
j
\end{array}\right)\left(\sum_{i=1}^{m} \mu_{i} t_{i}^{j}\right) t^{k-j},
$$

then $\quad \sum_{i=1}^{m} \mu_{i} v_{k, i}^{+}(t)=0$ if and only if $\sum_{j=0}^{k}\left(\begin{array}{c}k \\ j\end{array}\right)\left(\sum_{i=1}^{m} \mu_{i} t_{i}^{j}\right) t^{k-j}=0$. From Theorem 1, the set $\left\{t^{j}\right\}_{j=0}^{k}$ is linearly independent, it follows that $\sum_{i=1}^{m} \mu_{i} t_{i}^{j}=0$ for $j=0, \ldots k$. The matrix associated to this system is a Vandermonde type matrix. The rank of this matrix in $\min \{k+1, m\}$ and the result follows.

Polynomial weighted least squares fitting: Under the condition that $n<m$, we introduce the scalar product on $P_{n}$ defined by

$$
\langle p, q\rangle=\sum_{i=1}^{m} \omega_{i} p\left(t_{i}\right) q\left(t_{i}\right)
$$

for any pair of polynomials $p$ and $q$ in $P_{n}$. In this case (1) becomes

$$
F\left(p_{n}\right)=\left\|f-p_{n}\right\|^{2}
$$

where $\|\cdot\|$ is the norm on $P_{n}$ induced by the scalar product. For the $f_{i}$ 's we use the notation $f_{i}=f\left(t_{i}\right)(i=1, \ldots, m)$. It is well known that $p_{n}^{*}$ is unique and is characterized by the normal equations $\left\langle f-p_{n}^{*}, p_{n}\right\rangle=0$ for all $p_{n} \in P_{n}$.
In this setting, to simplify the computation of $p_{n}^{*}$, we can find a sequence of orthogonal polynomials by applying the Gram-Schmidt orthogonalization process to the standard basis $\left\{1, t, t^{2}, \ldots, t^{n}\right\}$ of $P_{n}$. These orthogonal polynomials are given by

$$
q_{0}(t)=1, q_{1}(t)=t-\alpha_{1}
$$

and for $j=2, \ldots, n$,

$$
q_{j}(t)=\left(t-\alpha_{j}\right) q_{j-1}(t)-\beta_{j} q_{j-2}(t)
$$

where

$$
\alpha_{j}=\frac{\left\langle t q_{j-1}, q_{j-1}\right\rangle}{\left\langle q_{j-1}, q_{j-1}\right\rangle} \quad(j=1,2, \ldots, n)
$$

and

$$
\beta_{j}=\frac{\left\langle t q_{j-1}, q_{j-2}\right\rangle}{\left\langle q_{j-2}, q_{j-2}\right\rangle} \quad(j=2,3, \ldots, n) .
$$

Hence the best $n$-degree least squares polynomial $p_{n}^{*}$ can be written as

$$
p_{n}^{*}(t)=\sum_{j=0}^{n} \gamma_{j}^{*} q_{j}(t)
$$

where

$$
\gamma_{j}^{*}=\frac{\left\langle f, q_{j}\right\rangle}{\left\langle q_{j}, q_{j}\right\rangle} \quad(j=0,1, \ldots, n) .
$$

The next two results will be useful for finding sufficient conditions for the existence of the WLSE for a 3-parametric exponential model ${ }^{[2]}$.

Theorem 3: $\quad\left\langle f-p_{n-1}^{*}, t^{n}\right\rangle=\gamma_{n}^{*}\left\|q_{n}\right\|^{2} \quad$ for $n=0, \ldots, m-1$.

Proof. For $n=0$ it is obvious because $p_{n-1}^{*}=0$. For $n>0$, since $q_{n}(t)=t^{n}+p_{n-1}(t)$ where $p_{n-1}(t)$ is a polynomial of degree $\leq n-1$, and

$$
p_{n}^{*}(t)=\gamma_{n}^{*} q_{n}(t)+p_{n-1}^{*}(t),
$$

we have

$$
\begin{aligned}
\gamma_{n}^{*}\left\|q_{n}\right\|^{2} & =\left\langle\gamma_{n}^{*} q_{n}, q_{n}\right\rangle \\
& =\left\langle p_{n}^{*}-p_{n-1}^{*}, q_{n}\right\rangle \\
& =\left\langle p_{n}^{*}-f, q_{n}\right\rangle+\left\langle f-p_{n-1}^{*}, q_{n}\right\rangle \\
& =\left\langle f-p_{n-1}^{*}, t^{n}+p_{n-1}\right\rangle \\
& =\left\langle f-p_{n-1}^{*}, t^{n}\right\rangle
\end{aligned}
$$


Theorem 4: If the $q_{j}$ 's are the orthogonal polynomials associated to $\left\{\left(\omega_{i}, t_{i}\right)\right\}_{i=1}^{m}$, the orthogonal polynomials $\tilde{q}_{j}$ 's associated to $\left\{\left(\omega_{i}, \tilde{t}_{i}=-t_{i}\right)\right\}_{i=1}^{m}$ are given by $\tilde{q}_{j}(t)=(-1)^{j} q_{j}(-t)$.

\section{POLYNOMIAL WEIGHTED LEAST SQUARES FITTING IN $I R^{m}$}

In the first part of this section we present the underlying subspaces of $I R^{m}$ related to the polynomial weighted least squares problem. In the second part we solve the problem using a projection onto a subspace of $I R^{m}$.

Vector spaces: Let $\left\{t_{i}\right\}_{i=1}^{m}$ be a set of $m$ distinct real numbers. For any positive integer $j$ let us define the vectors $\vec{t}_{j} \in I R^{m}$ by

$$
\vec{t}_{j}=\left(\begin{array}{c}
t_{1}^{j} \\
t_{2}^{j} \\
\vdots \\
t_{m}^{j}
\end{array}\right) \in I R^{m}
$$

For any positive integer $k$, we also define the vectors

$$
\vec{v}_{k, i}^{+}=\left(\vec{t}+t_{i} \overrightarrow{1}\right)^{k}=\sum_{j=0}^{k}\left(\begin{array}{c}
k \\
j
\end{array}\right) t_{i}^{j} \vec{t}^{k-j}
$$

for $i=1, \ldots, m$, and

$$
\vec{v}_{k, i}^{-}=\left(\vec{t}-t_{i} \overrightarrow{1}\right)^{k}=\sum_{j=0}^{k}(-1)^{j}\left(\begin{array}{c}
k \\
j
\end{array}\right) t_{i}^{j} \vec{t}^{k-j}
$$

for $i=1, \ldots, m$.

In this section we clarify the properties of the following vector spaces, in particular the dimension of the vector spaces,

$$
\begin{aligned}
T^{n} & =\operatorname{Lin}\left\{\vec{t}^{j} \mid j=0, \ldots, n\right\}, \\
V_{k}^{+} & =\operatorname{Lin}\left\{\vec{v}_{k, i}^{+} \mid i=1, \ldots, m\right\}, \\
V_{k}^{-} & =\operatorname{Lin}\left\{\vec{v}_{k, i}^{-} \mid i=1, \ldots, m\right\},
\end{aligned}
$$

for any integers $n$ and $k$ such that $n \geq 0$ and $0 \leq k \leq m-1$.

Theorem 5: $\operatorname{Let} T^{n}=\operatorname{Lin}\left\{\hat{t}^{j} \mid j=0, \ldots, n\right\} \subseteq I R^{m}$

(a) If $n<m$, the set $\left\{\hat{t}^{j}\right\}_{j=0}^{n}$ is linearly independent and $\operatorname{dim} T^{n}=n+1$. (b) If $n \geq m$, the set $\left\{\vec{t}^{j}\right\}_{j=0}^{n}$ is linearly dependent and $\operatorname{dim} T^{n}=m$.

Proof: We consider $\sum_{j=0}^{n} \lambda_{j} \vec{t}^{j}=0$. But the Vandermonde matrix $A_{m, n+1}=\left(\begin{array}{llll}\vec{t}^{0} & \vec{t}^{1} & \ldots & \vec{t}^{n}\end{array}\right)$ is of rank $n+1$ as long as $n<m$, and hence $\lambda_{j}=0$ for $j=0, \ldots, n$. If $n \geq m$ its rank is $m$ and there exits non zero solutions to the system. Hence the result follows because $T^{n} \subseteq I R^{m}$.

Remark 1: For any positive integer $l$, since $\vec{t}^{m+l} \in T^{m-1}=I R^{m}$, we have

$$
\vec{t}^{m+l}=\sum_{j=0}^{m-1} \lambda_{j}(l) \vec{t}^{j},
$$

where

$$
\vec{\lambda}(l)=\left(\begin{array}{c}
\lambda_{0}(l) \\
\lambda_{1}(l) \\
\vdots \\
\lambda_{m-1}(l)
\end{array}\right)=A_{m, m}^{-1} \vec{t}^{m+l}=A_{m, m}^{-1} \operatorname{diag}\left(\vec{t}^{m}\right) \vec{t}^{l},
$$

and

$$
\operatorname{diag}\left(\vec{t}^{m}\right)=\left(\begin{array}{cccc}
t_{1}^{m} & 0 & \ldots & 0 \\
0 & t_{2}^{m} & \ddots & \vdots \\
\ldots & \ddots & \ddots & 0 \\
0 & \ldots & 0 & t_{m}^{m}
\end{array}\right)
$$

Theorem 6: Let $k$ be any integer such that $0 \leq k \leq m-1$, and let $V_{k}^{+}$and $V_{k}^{-}$be defined by (7) and (8), then

and

$$
V_{k}^{+}=T^{k}=V_{k}^{-},
$$

$$
\operatorname{dim} V_{k}^{+}=k+1=\operatorname{dim} V_{k}^{-} .
$$

Proof. We prove the result for $V_{k}^{+}$only, the proof for $V_{k}^{-}$is identical. Since

$$
\begin{aligned}
\sum_{i=1}^{m} \mu_{i} \vec{v}_{k, i}^{+} & =\sum_{i=1}^{m} \mu_{i}\left(\sum_{j=0}^{k}\left(\begin{array}{l}
k \\
j
\end{array}\right) t_{i}^{j} \vec{t}^{k-j}\right) \\
& =\sum_{j=0}^{k}\left(\begin{array}{c}
k \\
j
\end{array}\right)\left(\sum_{i=1}^{m} \mu_{i} t_{i}^{j}\right) \vec{t}^{k-j},
\end{aligned}
$$


then $\quad \sum_{i=1}^{m} \mu_{i} \vec{v}_{k, i}^{+}=0$ if and only if $\sum_{j=0}^{k}\left(\begin{array}{l}k \\ j\end{array}\right)\left(\sum_{i=1}^{m} \mu_{i} t_{i}^{j}\right) \vec{t}^{k-j}=0$. From Theorem 5, the set $\left\{\vec{t}^{k-j}\right\}_{j=0}^{k}$ is linearly independent for $k<m$, it follows that $\sum_{i=1}^{m} \mu_{i} t_{i}^{j}=0$ for $j=0, \ldots k$. But this system of $k+1$ equations and $m$ unknowns has a unique solution only for $k=m-1$. Moreover the matrix associated to this system, $A_{m, k+1}^{T}$, is of rank $k+1$ for $k<m$. Hence $\operatorname{dim} V_{k}^{+}=k+1$.

For $k \geq m$ we have no clear result about the dimension of $V_{k}^{-}$and $V_{k}^{+}$as illustrated by the following example for $m=3$.

Example: Let $m=3$.

(a) For $V_{k}^{-}$, since we have

$$
\begin{aligned}
\operatorname{Det}\left(\vec{v}_{k, 1}^{-}, \vec{v}_{k, 2}^{-}, \vec{v}_{k, 3}^{-}\right)= & \left|\begin{array}{ccc}
0 & \left(t_{1}-t_{2}\right)^{k} & \left(t_{1}-t_{3}\right)^{k} \\
\left(t_{2}-t_{1}\right)^{k} & 0 & \left(t_{2}-t_{3}\right)^{k} \\
\left(t_{3}-t_{1}\right)^{k} & \left(t_{3}-t_{2}\right)^{k} & 0
\end{array}\right| \\
& =\left[1+(-1)^{k}\right]\left(t_{1}-t_{2}\right)^{k}\left(t_{2}-t_{3}\right)^{k}\left(t_{3}-t_{1}\right)^{k} \\
& =\left\{\begin{array}{ccc}
0 & \text { if } k \text { is odd }, \\
2\left(t_{1}-t_{2}\right)^{k}\left(t_{2}-t_{3}\right)^{k}\left(t_{3}-t_{1}\right)^{k} & \text { if } \quad k \text { is even, }
\end{array}\right.
\end{aligned}
$$

it follows that

$$
\operatorname{dim} V_{k}^{-}= \begin{cases}2 & \text { if } k \text { is odd } \\ 3 & \text { if } k \text { is even }\end{cases}
$$

(b) For $V_{k}^{+}$, we have

$$
\begin{aligned}
\operatorname{Det}\left(\vec{v}_{k, 1}^{+}, \vec{v}_{k, 2}^{+}, \vec{v}_{k, 3}^{+}\right)= & \left|\begin{array}{ccc}
\left(2 t_{1}\right)^{k} & \left(t_{1}+t_{2}\right)^{k} & \left(t_{1}+t_{3}\right)^{k} \\
\left(t_{2}+t_{1}\right)^{k} & \left(2 t_{2}\right)^{k} & \left(t_{2}+t_{3}\right)^{k} \\
\left(t_{3}+t_{1}\right)^{k} & \left(t_{3}+t_{2}\right)^{k} & \left(2 t_{3}\right)^{k}
\end{array}\right| \\
& =\left(8 t_{1} t_{2} t_{3}\right)^{k}+2\left(t_{1}+t_{2}\right)^{k}\left(t_{2}+t_{3}\right)^{k}\left(t_{3}+t_{1}\right)^{k} \\
& =-2^{k}\left[t_{1}^{k}\left(t_{2}+t_{3}\right)^{2 k}+t_{2}^{k}\left(t_{3}+t_{1}\right)^{2 k}+t_{3}^{k}\left(t_{1}+t_{2}\right)^{2 k}\right]
\end{aligned}
$$

This determinant can be 0 . Indeed for $t_{1}+t_{3}=0$ and $t_{2}=0$ the determinant is 0 for odd $k$. It follows that $\operatorname{dim} V_{k}^{+}$is 2 or 3 depending on the values of $t_{1}, t_{2}$ and $t_{3}$.

Remark 2: In ${ }^{[5]}$ it is asserted that $V_{2}^{-}$is of dimension $m$ which is clearly false except for $m=3$. As a consequence the proof given in ${ }^{[5]}$ for the existence of a WLSE for a 3-parametric exponential function is not correct. There are also errors in the proof of the existence of a WLSE in ${ }^{[6]}$.

Polynomial weighted least squares fitting: $\mathrm{We}$ introduce the scalar product on $I R^{m}$ defined by

$$
\langle\vec{u}, \vec{v}\rangle=\sum_{i=1}^{m} \omega_{i} u_{i} v_{i}
$$

for any pair of vectors $\vec{u}$ and $\vec{v}$ in $I R^{m}$

$$
\vec{u}=\left(\begin{array}{c}
u_{1} \\
u_{2} \\
\vdots \\
u_{m}
\end{array}\right) \text { and } \vec{v}=\left(\begin{array}{c}
v_{1} \\
v_{2} \\
\vdots \\
v_{m}
\end{array}\right)
$$

The norm on $I R^{m}$ induced by the scalar product is $\|\vec{u}\|=\langle\vec{u}, \vec{u}\rangle^{2}$. Then (1) becomes

where

$$
F\left(p_{n}\right)=\left\|\vec{f}-\vec{p}_{n}\right\|^{2}
$$

$$
\vec{p}_{n}=\sum_{j=0}^{n} \alpha_{j} \vec{t}^{j}, \quad \vec{t}^{j}=\left(\begin{array}{c}
t_{1}^{j} \\
t_{2}^{j} \\
\vdots \\
t_{m}^{j}
\end{array}\right), \quad \text { and } \quad \vec{f}=\left(\begin{array}{c}
f_{1} \\
f_{2} \\
\vdots \\
f_{m}
\end{array}\right) \text {. }
$$

The problem is to find the orthogonal projection of $\vec{f}$ on $T^{n}$. This projection is completely characterized by the normal equations $\left\langle\vec{f}-\vec{p}_{n}^{*}, \vec{p}_{n}\right\rangle=0$ for all $\vec{p}_{n} \in T^{n}$.

Again, to simplify the computation of $\vec{p}_{n}^{*}$, we can determine an orthogonal basis $\left\{\vec{q}_{j}\right\}_{j=0}^{n}$ for $T^{n}$ by applying the Gram-Schmidt process to its basis $\left\{\vec{t}^{j}\right\}_{j=0}^{n}$. We obtain

and for $j=2, \ldots, n$,

$$
\vec{q}_{0}=\overrightarrow{1}, \quad \vec{q}_{1}=\vec{t}-\alpha_{1} \overrightarrow{1}
$$

$$
\vec{q}_{j}=\left(\vec{t}-\alpha_{j} \overrightarrow{1}\right) \cdot \vec{q}_{j-1}-\beta_{j} \vec{q}_{j-2}
$$

where

$$
\alpha_{j}=\frac{\left\langle\vec{t} \cdot \vec{q}_{j-1}, \vec{q}_{j-1}\right\rangle}{\left\langle\vec{q}_{j-1}, \vec{q}_{j-1}\right\rangle} \quad(j=1,2,3, \ldots)
$$

and

$$
\beta_{j}=\frac{\left\langle\vec{t} \cdot \vec{q}_{j-1}, \vec{q}_{j-2}\right\rangle}{\left\langle\vec{q}_{j-2}, \vec{q}_{j-2}\right\rangle} \quad(j=2,3,4, \ldots) .
$$


In these identities, $\vec{u} \cdot \vec{v}$ is the coordinatewise multiplication of two vectors of $I R^{m}$ defined by

$$
\vec{u} \cdot \vec{v}=\left(\begin{array}{c}
u_{1} v_{1} \\
u_{2} v_{2} \\
\vdots \\
u_{m} v_{m}
\end{array}\right) .
$$

Let us observe that $\vec{q}_{j} \in T^{j}$ for $j=0, \ldots n$.

It follows that the projection is given by

$$
\vec{p}_{n}^{*}=\sum_{j=0}^{n} \gamma_{j}^{*} \vec{q}_{j}
$$

where

$$
\gamma_{j}^{*}=\frac{\left\langle\vec{f}, \vec{q}_{j}\right\rangle}{\left\langle\vec{q}_{j}, \vec{q}_{j}\right\rangle} \quad(j=0,1, \ldots, n)
$$

The next theorem is equivalent to Theorem 2.3.

Theorem 7: $\left\langle\vec{f}-\vec{p}_{n-1}^{*}, \vec{t}^{n}\right\rangle=\gamma_{n}^{*}\left\|\vec{q}_{n}\right\|^{2}$ for $n=0, \ldots, m-1$.

Proof. For $n=0$ we have $\vec{p}_{n-1}^{*}=\overrightarrow{0}$ and the result follows. For $n>0$, since $\vec{q}_{n}=\vec{t}^{n}+\vec{p}_{n-1}$ where $\vec{p}_{n-1}$ is a vector in $T^{n-1}$, and $\vec{p}_{n}^{*}=\gamma_{n}^{*} \vec{q}_{n}+\vec{p}_{n-1}^{*}$, we have

$$
\begin{aligned}
\gamma_{n}^{*}\left\|\vec{q}_{n}\right\|^{2} & =\left\langle\gamma_{n}^{*} \vec{q}_{n}, \vec{q}_{n}\right\rangle \\
& =\left\langle\vec{p}_{n}^{*}-\vec{p}_{n-1}^{*}, \vec{q}_{n}\right\rangle \\
& =\left\langle\vec{p}_{n}^{*}-\vec{f}, \vec{q}_{n}\right\rangle+\left\langle\vec{f}-\vec{p}_{n-1}^{*}, \vec{q}_{n}\right\rangle \\
& =\left\langle\vec{f}-\vec{p}_{n-1}^{*}, \vec{t}^{n}+\vec{p}_{n-1}\right\rangle \\
& =\left\langle\vec{f}-\vec{p}_{n-1}^{*}, \vec{t}^{n}\right\rangle .
\end{aligned}
$$

\section{CLASSIFICATION OF DATA}

Let $\left\{\left(\omega_{i}, t_{i}, f_{i}\right)\right\}_{i=1}^{m}$ be a set of $m$ data points. If we use a discrete least squares polynomial to fit the data with the orthogonal basis $\left\{q_{j}\right\}_{j=0}^{n}$, the coefficients of $p_{n}^{*}$ with respect to its expansion (5) or (9) suggest the following classification of the data.

Definition 1: The data $\left\{\left(\omega_{i}, t_{i}, f_{i}\right)\right\}_{i=1}^{m}$ are said to be:

(i) essentially stationary if $\gamma_{1}^{*}=0$;

(ii) essentially increasing, respectively decreasing, if $\gamma_{1}^{*}>0$, respectively $\gamma_{1}^{*}<0$; (iii) essentially linear if $\gamma_{2}^{*}=0$;

(iv) essentially convex, respectively concave, if $\gamma_{2}^{*}>0$, respectively $\gamma_{2}^{*}<0$.

Let us note that we could continue the classification with the higher order coefficients $\gamma_{n}^{*}$ for $n=3, \ldots, m-1$. this basic classification could help to find more realistic or complex fitting to the data with nonlinear function (see $[4,5,6,2]$ for an exponential functions).

Finally if we apply symmetric transformations to the data we obtain the following result.

Theorem 8: Effect of symmetric transformations on the data.

(a) If the $\left\{\left(\omega_{i}, t_{i}, f_{i}\right)\right\}_{i=1}^{m}$ are essentially increasing, resp. decreasing, then the data $\left\{\left(\omega_{i},-t_{i}, f_{i}\right)\right\}_{i=1}^{m}$ are essentially decreasing, resp. increasing. The stationarity, linearity, and concavity or convexity properties are not modified by this transform.

(b) If the data $\left\{\left(\omega_{i}, t_{i}, f_{i}\right)\right\}_{i=1}^{m}$ are essentially increasing, resp. decreasing, and essentially convex, resp. concave, then the data $\left\{\left(\omega_{i}, t_{i},-f_{i}\right)\right\}_{i=1}^{m}$ are essentially decreasing, resp. increasing, and essentially concave, resp. convex. The stationarity and linearity properties are not modified by this transform.

\section{CONCLUSION}

We have revisited the polynomial weighted least squares analysis. Doing so we have specified the dimension of three vector subspaces of $P$ (Theorem 1 and Theorem 2) and of $I R^{m}$ (Theorem 5 and Theorem 6) used for solving this problem. We also have established a property (Theorem 3 and Theorem 7) and suggested a classification of data (Definition 1) which will play a role in finding sufficient conditions for the existence of a WLSE for a 3-parametric exponential $\operatorname{model}^{[2]}$.

\section{ACKNOWLEDGMENTS}

This work has been supported by a NSERC (Natural Sciences and Engineering Research Council of Canada) individual discovery grant for the first author 


\section{REFERENCES}

1. S.D. Conte and C. de Boor, 1980. Elementary Numerical Analysis: An Algorithmic Approach, McGraw-Hill, New York.

2. F. Dubeau and Y. Mir, 2007. Existence of optimal weighted least squares estimate for 3-parametric exponential model, Communications in Statistics Theory and Methods, to appear.

3. G.E. Forsythe, 1956. Generation and use of orthogonal polynomials for data-fitting with digital computer, J. SIAM, 5, 74-78.

4. D. Jukic and R. Scitovski, 1997. Existence of optimal solution for exponential model by least squares, Journal of Computational and Applied Mathematics, 78, 317-328.
5. D. Jukic and R. Scitovski, 2000. The best least squares approximation problem for a 3-parametric exponential regression model, ANZIAM J., 42, 254-266.

6. D. Jukic, 2004 A necessary and sufficient criteria for the existence of the least squares estimate for a 3-parametric exponential function, Applied Mathematics and Computation, 147, 1-17.

7. C.L. Lawson and R.J. Hanson, 1995. Solving Least Squares Problems, SIAM, Philadelphia.

8. L.F. Shampine, 1975. Discrete least squares polynomial fits, Communications of the ACM, 18, 179-180.

9. L.F. Shampine and R.C. Allen, 1973. Numerical Computing: An introduction, Saunders, Philadelphia. 